Kubandt, Melanie

\title{
Ansprüche an ein geschlechtergerechtes, professionelles Handeln im Elementarbereich. Ethnographische Perspektiven auf Ungewissheiten, Komplexitäten und Grenzen im pädagogischen Alltag
}

Baar, Robert [Hrsg.]; Hartmann, Jutta [Hrsg.]; Kampshoff, Marita [Hrsg.]: Geschlechterreflektierte Professionalisierung. Geschlecht und Professionalität in pädagogischen Berufen. Opladen ; Berlin ; Toronto : Verlag Barbara Budrich 2019, S. 121-133. - (Jahrbuch erziehungswissenschaftliche Geschlechterforschung; 15)

Quellenangabe/ Reference:

Kubandt, Melanie: Ansprüche an ein geschlechtergerechtes, professionelles Handeln im Elementarbereich. Ethnographische Perspektiven auf Ungewissheiten, Komplexitäten und Grenzen im pädagogischen Alltag - In: Baar, Robert [Hrsg.]; Hartmann, Jutta [Hrsg.]; Kampshoff, Marita [Hrsg.]: Geschlechterreflektierte Professionalisierung. Geschlecht und Professionalität in pädagogischen Berufen. Opladen ; Berlin ; Toronto : Verlag Barbara Budrich 2019, S. 121-133 - URN: urn:nbn:de:0111-pedocs-219052 - DOI: 10.25656/01:21905

https://nbn-resolving.org/urn:nbn:de:0111-pedocs-219052 https://doi.org/10.25656/01:21905

in Kooperation mit / in cooperation with:

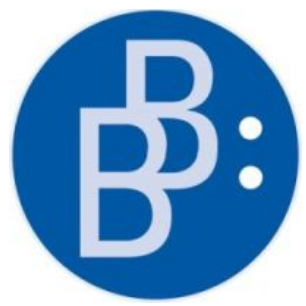

https://www.budrich.de

\section{Nutzungsbedingungen}

Gewährt wird ein nicht exklusives, nicht übertragbares, persönliches und beschränktes Recht auf Nutzung dieses Dokuments. Dieses Dokument ist ausschließlich für den persönlichen, nicht-kommerziellen Gebrauch bestimmt. Die Nutzung stellt keine Übertragung des Eigentumsrechts an diesem Dokument dar und gilt vorbehaltlich der folgenden Einschränkungen: Auf sämtlichen Kopien dieses Dokuments müssen alle Urheberrechtshinweise und sonstigen Hinweise auf gesetzlichen Schutz beibehalten werden. Sie dürfen dieses Dokument nicht in irgendeiner Weise abändern, noch dürfen Sie dieses Dokument für öffentliche oder kommerzielle Zwecke vervielfältigen, öffentlich ausstellen, aufführen, vertreiben oder anderweitig nutzen.

Mit der Verwendung dieses Dokuments erkennen Sie die Nutzungsbedingungen an.

\section{Terms of use}

We grant a non-exclusive, non-transferable, individual and limited right to using this document.

This document is solely intended for your personal, non-commercial use. Use of this document does not include any transfer of property rights and it is conditional to the following limitations: All of the copies of this documents must retain all copyright information and other information regarding legal protection. You are not allowed to alter this document in any way, to copy it for public or commercial purposes, to exhibit the document in public, to perform, distribute or otherwise use the document in public.

By using this particular document, you accept the above-stated conditions of use.

\section{Kontakt / Contact:}

peDOCS

DIPF | Leibniz-Institut für Bildungsforschung und Bildungsinformation Informationszentrum (IZ) Bildung

E-Mail: pedocs@dipf.de

Internet: www.pedocs.de

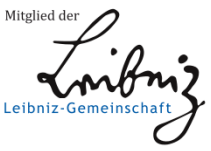


Robert Baar

Jutta Hartmann

Marita Kampshoff (Hrsg.)

\section{$15 / 2019$}

Jahrbuch erziehungswissenschaftliche Geschlechterforschung

Geschlechterreflektierte Professionalisierung Geschlecht und Professionalität in pädagogischen Berufen 


\section{Robert Baar}

Jutta Hartmann

Marita Kampshoff (Hrsg.)

\section{Geschlechterreflektierte Professionalisierung - Geschlecht und Professionalität in pädagogischen Berufen}

Verlag Barbara Budrich Opladen • Berlin • Toronto 2019 
Bibliografische Information der Deutschen Nationalbibliothek

Die Deutsche Nationalbibliothek verzeichnet diese Publikation in der Deutschen

Nationalbibliografie; detaillierte bibliografische Daten sind im Internet über

http://dnb.d-nb.de abrufbar.

Gedruckt auf säurefreiem und alterungsbeständigem Papier.

Alle Rechte vorbehalten.

(C) 2019 Verlag Barbara Budrich, Opladen, Berlin \& Toronto

www.budrich-verlag.de

ISBN 978-3-8474-2277-8 (Paperback)

eISBN 978-3-8474-1310-3 (eBook)

Das Werk einschließlich aller seiner Teile ist urheberrechtlich geschützt. Jede Verwertung außerhalb der engen Grenzen des Urheberrechtsgesetzes ist ohne Zustimmung des Verlages unzulässig und strafbar. Das gilt insbesondere für Vervielfältigungen, Übersetzungen, Mikroverfilmungen und die Einspeicherung und Verarbeitung in elektronischen Systemen.

Typographisches Lektorat: Anja Borkam, Jena

Umschlaggestaltung: disegno visuelle kommunikation, Wuppertal - www.disenjo.de Druck: paper \& tinta, Warschau

Printed in Europe 


\section{Inhalt}

\section{Einleitung}

Robert Baar, Jutta Hartmann \& Marita Kampshoff

Vorwort. 7

Robert Baar, Jürgen Budde, Marita Kampshoff \& Astrid Messerschmidt Redaktion des Jahrbuchs erziehungswissenschaftliche

Geschlechterforschung

Von der Frauen- und Geschlechterforschung in der

Erziehungswissenschaft zur erziehungswissenschaftlichen

Geschlechterforschung....

Maja S. Maier

Erziehungswissenschaftliche Geschlechterforschung?

Ein Essay zu Verhältnisbestimmung und Forschungsprogrammatik 15

Robert Baar, Jutta Hartmann \& Marita Kampshoff

Geschlechterreflektierte Professionalisierung - Geschlecht und

Professionalität in pädagogischen Berufen. Eine Einführung

\section{Vielfältige Lebensweisen im Fokus von Professionalität}

Florian Cristobal Klenk

Interdependente Geschlechtervielfalt als un/be/deutende Anforderung an pädagogische Professionalität

Mart Busche \& Uli Streib-Brzič

Die Entwicklung heteronormativitätskritischer Professionalität in

Reflexions-Workshops - Zur Verbindung von pädagogischem

Erfahrungswissen und wissenschaftlichem Erkenntniswissen im Kontext

von Praxisforschung

Carolin Vierneisel \& Johannes Nitschke

(De-)Professionalisierungstendenzen?! Vielfalts*sensible Bildung im

Lehramtsstudium. 


\section{Geschlechterreflektierte Professionalität im Elementarbereich}

\section{Melanie Kubandt}

Ansprüche an ein geschlechtergerechtes, professionelles Handeln im Elementarbereich - Ethnographische Perspektiven auf Ungewissheiten,

Komplexitäten und Grenzen im pädagogischen Alltag

Susann Fegter, Anna Hontschik, Eszter Kadar, Kim-Patrick Sabla \& Maxine Saborowski

Bezüge auf Familie als Moment der Vergeschlechtlichung pädagogischer Professionalität: Diskursanalytische Perspektiven auf Äußerungen in Gruppendiskussionen mit Kita-Teams

\section{Herausforderungen sexualpädagogischer Professionalisierung}

\section{Anja Eichhorn}

Doing Sexual Agency: Sexuelle Handlungsfähigkeit sexuell

missbrauchter jugendlicher Mädchen in der stationären Jugendhilfe

Marion Thuswald

Geschlechterreflektierte sexuelle Bildung?

Heteronormativität und Verletzbarkeit als Herausforderungen

sexualpädagogischer Professionalisierung

\section{Rezension}

Marina Dangelat, Frauke Grenz \& Christine Thon

Rezension zu: Gesicht Zeigen! (2017) (Hrsg.): „Weiße können nicht rappen“. Das Positionierungsspiel gegen Vorurteile und Klischees und zu: Wedl, Juliette (2018): Identitätenlotto. Ein Spiel quer durchs Leben ... 183

Verzeichnis der Autor_innen 191 


\section{Ansprüche an ein geschlechtergerechtes, professionelles Handeln im Elementarbereich - Ethnographische Perspektiven auf Ungewissheiten, Komplexitäten und Grenzen im pädagogischen Alltag}

\section{Hintergrund: Die Besonderheit professionellen pädagogischen Handelns im Elementarbereich}

Professionsentwicklungen in der frühkindlichen Bildung, Betreuung und Erziehung in Deutschland sind im europäischen Vergleich ein Spezifikum und deswegen nur schwer in all ihren Facetten zu beschreiben. Denn die Berufsbildungsgänge werden primär im vollzeitschulischen Modus in öffentlichen und trägergeführten Fachschulen bzw. -akademien realisiert, sind im Hochschulsystem ebenfalls in Länderregie entstanden und haben sich außerhalb und neben dem Berufsausbildungssystem dualer Berufe von Wirtschaft, Handwerk und Industrie etabliert (vgl. Karsten 2017). Rabe-Kleberg (1993) verweist wiederholt auf Besonderheiten dieses spezifischen Berufskontextes, der mit Herausforderungen an professionelles Handeln verbunden ist, da die Tätigkeiten im elementarpädagogischen Feld immer personenbezogen und die Abläufe nicht vorhersehbar sind. Auch Hoffmann et al. (2014) betonen die Herausforderungen professionellen Handelns im Elementarbereich und kritisieren einen gängigen Fokus auf die Erzeugung evidenzbasierten Wissens. Denn durch die Konjunktur der empirischen Bildungsforschung und großer Leistungsvergleichsstudien seit PISA zeigt sich im Elementarbereich die Tendenz, dass „die Frage nach einer professionellen pädagogischen Praxis aktuell mit empirisch gesichertem Wissen darüber, was wirkt", verbunden wird, ,,angeregt durch eine verstärkte Outputorientierung im Sinne nachweisbarer Leistungen" (ebd.: 47f.). Eine solche Perspektiveinnahme gehe jedoch mit blinden Flecken einher, nämlich dann, wenn dadurch „Aspekte wie Ungewissheit, Kontextgebundenheit, Komplexität und die Frage danach, welche pädagogischen Ziele wünschenswert bzw. angemessen sind“ ausgespart werden, wenngleich sie „ein wesentliches Moment professionellen pädagogischen Handelns darstellen“ (ebd.: 48). Problematisch ist ein solches Verständnis, wenn dies mit Vorstellungen von erwerbbaren Kompetenzen verbunden ist und auf Machbar- und 
v.a. Planbarkeit professionellen Handelns verweist. Riegel (2013) hat am Beispiel des professionellen Umgangs mit Differenzlinien auf Schwierigkeiten hingewiesen, die mit einem einseitigen Kompetenzverständnis und verkürzten Perspektiven auf Herausforderungen einhergehen. Herausfordernd wird es dann, wenn ,der Umgang mit Heterogenität als eine wichtige Qualifikation für Heranwachsende in globalisierten und internationalisierten Lebensverhältnissen (...) betrachtet“ wird. Denn ein verkürztes „Kompetenz-Verständnis bleibt weitgehend in der Logik der individualisierten Aneignung von gesellschaftlich relevanten Kompetenzen und deren Verwertung gefangen“, zudem ist eine solche Vorstellung ,eng mit der Ausbildung von Humankapital verknüpft, mit dem Ziel eines möglichst effektiven ,managings' von Unterschieden“" (ebd.: 188).

Vor diesem Hintergrund wird im Folgenden am Beispiel des Anspruchs einer Realisierung von Geschlechtergerechtigkeit in Kindertageseinrichtungen zur Diskussion gestellt, inwiefern eine ethnographische Perspektiveinnahme auf Prozesse des doing gender sowohl empirische, pädagogische als auch theoretische Einsichten zu generieren vermag, um einen Beitrag für eine geschlechterreflektierende Professionalisierungsdebatte auch abseits von Wirksamkeitsanforderungen zu offerieren (vgl. hierzu auch Kubandt 2018). Auf Basis empirischer Erkenntnisse eines ethnographischen Projekts zu doing gender in der Kindertageseinrichtung werden daher die Potenziale einer ethnographischen Perspektiveinnahme herausgearbeitet und zugleich die Grenzen, blinden Flecke und Paradoxien der Ansprüche an eine geschlechterreflektierende professionelle Praxis diskutiert.

\section{Geschlechtergerechtes Handeln als pädagogische Querschnittsaufgabe: Geschlecht als Potenzial vs. Geschlecht als Ungleichheitsdimension}

In zahlreichen frühpädagogischen Bildungs-, Erziehungs- und Orientierungsplänen wird die Realisierung von Geschlechtergerechtigkeit ausdrücklich als Querschnittsaufgabe der pädagogischen Arbeit in Kindertageseinrichtungen hervorgehoben (vgl. Kubandt 2015; Meyer 2015). Vor der normativen Folie von Gerechtigkeit findet sich in den frühpädagogischen Bildungs-, Erziehungs- und Orientierungsplänen zum einen die Tendenz, Geschlecht als potenzielle Ungleichheitsdimension zu postulieren, die soziale Ungerechtigkeit und Benachteiligungen mit bedingen kann. Deutlich häufiger wird Geschlecht allerdings positiv konnotiert zum Thema. In der Regel erfolgt die Thematisie- 
rung von Geschlecht gemeinsam mit anderen Differenzlinien als zu berücksichtigende und für die kindliche Entwicklung wesentliche Identitätsdimension (vgl. Meyer 2015; 2018). Im Niedersächsischen Orientierungsplan für Bildung und Erziehung im Elementarbereich (2005) heißt es exemplarisch: „Mädchen und Jungen müssen ihre eigene Geschlechtsidentität entwickeln können, ohne durch stereotype Sichtweisen und Zuschreibungen in ihren Erfahrungsmöglichkeiten eingeschränkt zu werden. Jungen und Mädchen erhalten gleiche Chancen, die Aufmerksamkeit und Unterstützung der Fachkräfte zu erlangen“" (ebd.: 10). Unabhängig davon, ob Positiv- oder Negativmarkierungen von Geschlecht erfolgen, werden Anforderungen an Fachkräfte formuliert. Vor allem die Fähigkeit zur Selbstreflexion wird wiederholt als zentrales Mittel betont, um Geschlechtergerechtigkeit in Kindertageseinrichtungen realisieren zu können.

Wissenschaftlich dominiert aktuell eher eine negativ markierte Sichtweise auf Differenzlinien, wie sie beispielsweise für machtkritische Ungleichheitsansätze typisch ist. Während folglich in praxisnahen, bildungspolitischen $\mathrm{Zu}-$ sammenhängen affirmative Perspektiven auf Geschlecht überwiegen, finden sind in wissenschaftlichen Kontexten häufiger machtkritische Differenzpositionen (vgl. u.a. Diehm et al. 2013; Machold 2015). Auch wenn affirmative und machtkritische Perspektiveinnahmen prinzipiell gegenläufig sind, vereint sie die gemeinsame, übergeordnete Frage nach Gerechtigkeit. Zudem firmieren sie mal mehr mal weniger explizit um die Frage, wie Geschlecht angemessen professionell im pädagogischen Alltag verhandelt werden sollte. Interessant ist hierbei, dass normativ-präskriptive Setzungen zu Geschlecht auf Ebene von bildungspolitischen Vorgaben und/oder auf Ebene von Studienanlagen folglich sowohl den Ausgangspunkt als auch das Ziel gängiger Positionierungen bestimmen. Unberücksichtigt bleibt gerade in praxisorientierten Diskussionskontexten jedoch der Sachverhalt, dass Geschlechtergerechtigkeit je nach inhaltlicher Ausrichtung je unterschiedlich gedacht werden kann und die teils inflationär propagierten Ansprüche an ein geschlechtergerechtes, professionelles Handeln inhaltlich(er) zu konturieren sind.

\section{Die inhaltliche Kontingenz von Geschlechter- gerechtigkeit als kritisches Moment professionellen Handelns in Kindertageseinrichtungen}

Nimmt man bildungspolitische Debatten in den Blick, so begegnet man unterschiedlichen Begrifflichkeiten, die im Zusammenhang mit Geschlechtergerechtigkeit Verwendung finden. Dies zeigt bereits ein Blick in die Bildungs-, 
Erziehungs- und Orientierungspläne der Bundesländer. Geschlecht wird jeweils mit Begrifflichkeiten wie geschlechtersensibel (vgl. Baden-Württemberg, Bayern, Rheinland-Pfalz, Nordrhein-Westfalen, Sachsen), genderbewusst (vgl. Niedersachsen, Schleswig-Holstein), geschlechterbewusst (vgl. Sachsen, Hamburg, Nordrhein-Westfalen) und/oder genderorientiert (vgl. Schleswig-Holstein) verknüpft. Dabei wird nicht immer deutlich, ob die Begriffe synonym verwendet oder mit unterschiedlichen Vorstellungen von $\mathrm{Ge}$ schlecht verbunden sind (vgl. ausf. Kubandt 2016). Bereits die Vielzahl der in den Debatten kursierenden Begrifflichkeiten verweist auf die Komplexität der Ansprüche bei gleichzeitiger Uneindeutigkeit der konkreten Anforderungen an pädagogische Fachkräfte. Rendtorff (2017) hat diesen blinden Fleck für schulpädagogische Debatten um Geschlechtergerechtigkeit problematisiert und kritisiert an dem dort weit verbreiteten Begriff gendersensibel folgendes:

„[M] it dem Begriff alleine kommen wir nicht weiter. Indem er so tut, als wüsste er (oder: als sei klar), was er bedeutet, verhindert er sogar die Auseinandersetzung mit dem Sachverhalt, um den es eigentlich geht $[\ldots]$ Es würde auch nicht helfen, wenn wir den Ausdruck, gendersensibel' durch benachbarte Begriffe ersetzen würden, etwa ,geschlechtergerecht' oder ,genderbewusst', denn das Problem der Breite unterschiedlicher und konkreter Auslegungsmöglichkeiten bleibt auch bei diesen unverändert bestehen“ (ebd.: 18).

Da unter Begrifflichkeiten, wie z.B. Gendersensibilität etwas je Unterschiedliches, zum Teil gar gegensätzliche Vorstellungen von Gerechtigkeit verstanden werden können, sind die inhaltliche Ausdifferenzierung von Geschlechtergerechtigkeit und entsprechendes professionelles Handeln prinzipiell kontingent zu denken: manche Positionen argumentieren geschlechtergerechtes Handeln im Sinne einer Vermeidung geschlechtlicher Zuschreibungen, andere Positionen argumentieren hingegen mit der Betonung von Differenzen bzw. der Berücksichtigung von spezifischen Bedürfnissen und/oder Fähigkeiten. Folglich können die gängigen Begrifflichkeiten (wie u.a. gendersensibel, gendergerecht, geschlechterbewusst, und/oder gendersensibel) sowohl für Strategien stehen, die Geschlechterunterschiede betonen als auch marginalisieren (vgl. Kubandt 2016). Auch Rendtorff (2017) verweist auf diesen Sachverhalt und hebt hervor, dass

„[b]eide pädagogischen Strategien (...) also mit demselben Wort/Begriff umschrieben werden [könnten], obgleich sie sich diametral widersprechen. Wo sich die erste die geschlechterbezogenen Etikettierungen zunutze machen will und dadurch die Identifizierung mit Geschlechterzuschreibungen verstärkt, will die andere diese Zuschreibungen negieren, ihnen gerade nicht entgegenkommen, sie dadurch abmildern und sozusagen, verflüssigen." (ebd.: 19).

Knapp (2001) verweist daher zurecht darauf, dass immer, wenn Geschlecht in den Fokus genommen wird, vorab geklärt werden müsse, was damit gemeint ist: 
„Meint die Rede von Geschlecht, Geschlechterdifferenz als Eigenschafts- und Identitätskategorie, zielt sie auf Geschlechterbeziehungen als Relationen und Formen des Austauschs zwischen Männern und Frauen; auf Geschlechterordnungen als symbolisch-kulturelle Klassifikations- und Regulationssysteme oder auf Geschlechterverhältnisse als soziostrukturelle Organisationsform des Verhältnisses zwischen den Genus-Gruppen?““ (ebd.: 79). ${ }^{1}$

In Kubandt (2016) wird zudem auf die inhaltliche Kontingenz von Geschlechtergerechtigkeit als eine Herausforderung in elementarpädagogischen Geschlechterdebatten hingewiesen:

„[E]ine inhaltliche Ausdifferenzierung von Chancengleichheit und Gerechtigkeit ist einerseits mit schwierigen ethischen Fragen verbunden, die selbst auf theoretischer Ebene kaum $\mathrm{zu}$ beantworten sind und andererseits notwendigerweise mit spezifischen Entscheidungen verknüpft, die stets kontextabhängig und standortgebunden sind. Entsprechende Leerstellen und Herausforderungen müssten (...) jedoch zumindest benannt bzw. übergeordnet diskutiert werden. So wie Geschlecht derzeit frühpädagogisch für Kindertageseinrichtungen verhandelt wird, werden die mit einer geforderten Umsetzung von Geschlechtergerechtigkeit einhergehenden Herausforderungen allerdings eher marginalisiert bzw. vernachlässigt"“ (ebd.: 296).

In diesem Zusammenhang wird Geschlechtergerechtigkeit daher als ein „Konstrukt zwischen vermeintlicher Eindeutigkeit und unvermeidlicher Leerstelle“" (ebd.: 291) bezeichnet. Ohne dass folglich klar ist, was jeweils gemeint ist, zeigt sich bildungspolitisch unter verschiedensten Begrifflichkeiten die Anforderung, dass professionelles Handeln in Kindertageseinrichtungen die Verwirklichung von Geschlechtergerechtigkeit als Querschnittsaufgabe zu berücksichtigen habe. Es ist leicht vorstellbar, dass die Deutungsoffenheit der Anforderungen im Zusammenhang mit den Ansprüchen an ein geschlechtergerechtes professionelles Handeln in Kindertageseinrichtungen eine komplexe Herausforderung für pädagogische Fachkräfte darstellt. Zur Veranschaulichung dessen greife ich im Folgenden auf empirische Ergebnisse aus einem ethnographischen Projekt zu doing gender in der Kindertageseinrichtung zurück, das im Zeitraum von vierzehn Monaten in einer niedersächsischen Kindertageseinrichtung als teilnehmende Beobachtung durchgeführt wurde (vgl. Kubandt 2016). Die am ethnomethodologischen Paradigma ausgerichtete Fragestellung lautete, wie in der Einrichtung Geschlecht von den Akteur*innen (Kinder, Eltern, Fachkräfte) im pädagogischen Alltag konstruiert wird. Die folgenden Praxisbeispiele zu Geschlechterkonstruktionen von pädagogischen Fachkräften sollen aufzeigen, welche Unsicherheiten und blinden Flecke sich im pädagogischen Alltag vor der Folie des Anspruchs, geschlechtergerecht zu handeln, ergeben (können).

1 Auch wenn Knapp (2001) für empirische Perspektiven auf dieses Problem verweist, lässt es sich auch auf pädagogische Praxis übertragen, wenn es heißt: was verstehen wir eigentlich unter Geschlecht im pädagogischen Alltag? 


\section{Denn sie wissen nicht wie sie tun, was sie tun - ethnographische Geschlechterperspektiven auf pädagogisches Handeln in der Kindertageseinrichtung}

An die Ethnomethodologie angelehnte Forschungen zu doing gender gehen davon aus, ,dass Teilnehmern ihre alltagsweltlichen Konstruktionen nicht (notwendig) reflexiv oder diskursiv verfügbar sind, sondern sie verfügen über jene Form von impliziten Wissen oder, tacit knowledge': Die Teilnehmer wissen wie es geht, sich in der eigenen Kultur kompetent zu bewegen, aber sie wissen es nicht zu erklären - deswegen haben Beobachtungsverfahren Priorität" (Kelle 2004: 637). Hier setzte die genannte Studie an, in der pädagogische Praxis in einer Kindertageseinrichtung im Hinblick auf unterschiedliche Relevanzsetzungen von Geschlecht ethnographisch in den Blick genommen wurde. Ein zentrales Ergebnis der Studie war eine von den pädagogischen Fachkräften selbst propagierte geschlechterneutrale Haltung, die sich u.a. in der von ihnen inflationär wiederholten Aussage ,wir behandeln ja alle gleich“ niederschlug. Gleichzeitig herrschte Unsicherheit auf Seiten der Fachkräfte, ob ihre Haltung fachlich gut und angemessen sei. So wurde ich als Forscherin wiederholt von diversen Fachkräften gefragt, ob denn ein geschlechterneutraler Umgang aus meiner Sicht pädagogisch richtig sei. Darauf, dass eine vermeintlich geschlechterneutrale Haltung eher mit Unsicherheiten verbunden ist und nicht primär auf fachlicher Überzeugung beruht, verweist ein Protokollbeispiel aus einem Tür-und-Angel-Gespräch in der Kindertageseinrichtung. Darin unterhalte ich mich mit einer Fachkraft und der Leiterin der Einrichtung:

Die Fachkraft Steffie erzählt, sie würde im Alltag der Kita sehr viel reflektieren, gerade auch im Hinblick auf geschlechtliche Zuschreibungen. Sie sagt: „Aber man sagt/tut manchmal eben auch Dinge, die evtl. nicht korrekt sind, aber ich versuche immer alle gleich zu behandeln und geschlechterneutral zu agieren!" Ich hebe hervor, dass es mir schon ein paar Mal aufgefallen sei, dass es den Fachkräften in der Einrichtung offenbar wichtig ist, Unterschiede zwischen Jungen und Mädchen zu vermeiden und einen neutralen Umgang zu betonen. Die Leiterin Sabine fragt daraufhin etwas verunsichert: ,Aha, und ist das jetzt dann ein Kompliment, also ist das jetzt aus deiner Sicht gut oder schlecht, wenn wir die Kinder neutral behandeln?"2

Es konnte rekonstruiert werden, dass die Fachkräfte häufig unsicher waren, wann und ob geschlechtliche Unterschiede problematisch sind und wann und wie pädagogisch anzuerkennen. Des Weiteren wurde nachgezeichnet, dass es den pädagogischen Fachkräften offenbar dann leichter fiel Stereotypisierungen $\mathrm{zu}$ vermeiden, wenn es sich um planbare Angebote im pädagogischen Alltag handelte (vgl. hierzu ausführlicher Kubandt 2015). Gleichzeitig zeigten sich

Vgl. für eine ausführlicherer Darstellung der Szenen Kubandt 2016. 
trotz des eigenen Anspruchs ,alle Kinder gleich zu behandeln“ gerade in Alltagsinteraktionen Stereotypisierungen, die den Fachkräften selbst jedoch in der Regel nicht bewusst waren. Vielmehr konstruierten die Fachkräfte ihre eigenen geschlechtlichen Zuschreibungen als Relevanzsetzungen der Kinder, die sie aus ihrer Sicht lediglich beobachteten. Anhand des nachfolgenden Materialauszugs aus einem Beobachtungsprotokoll wird dies exemplarisch rekonstruierbar:

Während Sid, Timm und Lukas mit mir im Gruppenraum spielen, werden diese auf diverse Schmuckstücke (Ketten, Armreifen, Ohrringe, Ringe) aufmerksam, die auf einem kleinen Tisch liegen und von denen keiner von uns weiß, woher diese stammen. Neben uns sitzt die Fachkraft Katrin und malt gemeinsam mit Lisa an einem Bild mit Herbstlaub. Timm, Sid und Lukas nehmen mehrere Schmuckstücke und pieksen mich damit, ketten mich damit an und sagen, sie würden mich jetzt verarzten bzw. Blut abnehmen, es müsse dabei etwas wehtun und pieksen. Ich spiele mit und lasse mich in der nächsten Viertelstunde als Patientin mithilfe des Schmucks auf diverse Arten verarzten. Nach einer Weile lassen die Jungen ab von dem Schmuck, gehen rüber in den Mattenraum und fragen, ob ich mitkommen will. Auf meinem Weg in den Mattenraum fragt mich Katrin, ob ich den Schmuck in die Kita mitgebracht hätte. Ich verneine und sage, ich wüsste auch nicht, woher dieser stamme. Daraufhin sagt sie: „Ich dachte schon, wie cool für dich mit den Jungs und dem Schmuck, aber jetzt haben sie ja nicht wirklich mit dem Schmuck gespielt, es war also doch nicht so für dein Projekt geeignet gewesen.“

Der Sequenzausschnitt beschreibt ein gemeinsames Spiel von Sid, Timm, Lukas und mir, bei dem diese zufällig herumliegende Schmuckstücke zweckentfremden, um mich zu verarzten. Im weiteren Verlauf der Sequenz kommentiert die Fachkraft Katrin diese Szenerie. Zum einen verweist ihre Frage, ob der Schmuck von mir sei darauf, dass sie offenbar vermutet, ich hätte Schmuck mitgebracht, um eventuell zu sehen, wie die Kinder darauf reagieren bzw. mit diesem spielen würden. Durch ihre Kommentierung Ich dachte schon, wie cool für dich mit den Jungs und dem Schmuck, aber jetzt haben sie ja nicht wirklich mit dem Schmuck gespielt, es war also doch nicht so geeignet gewesen scheint sie in Anlehnung an meinen ihr bekannten Forschungsfokus Geschlecht eine Opposition zwischen den drei Jungen und dem vermeintlich weiblich konnotierten Schmuck zu thematisieren. In dieser Logik ist auch ihre Frage verständlich, ob der Schmuck von mir in die Einrichtung mitgebracht worden sei, da sie augenscheinlich davon ausgegangen ist, dass ich im Sinne eines wissenschaftlichen Experiments mit dem Schmuck etwas vermeintlich stereotyp Weibliches aktiv in die Einrichtung mitgebracht habe, um zu sehen, wie sich die Kinder damit beschäftigen. Interessant ist die veränderte Wertung in ihrer Aussage: zunächst konnotiert sie die Opposition Jungs und Schmuck mit ihrer Kommentierung wie cool für dich positiv. Dadurch, dass die Jungen aus ihrer Sicht jedoch anders als von ihr erwartet mit dem Schmuck gespielt haben, wird die ursprüngliche positive Wertung durch ihre Aussage aber jetzt haben sie ja nicht wirklich mit dem Schmuck gespielt, es war also doch nicht so geeignet gewesen dann jedoch eher negativ formuliert. Es scheint, als ob die Fachkraft 
eine implizite Vorannahme hatte, wie die Jungen auf den Schmuck reagieren würden und was dabei spannend für mich gewesen wäre. Allerdings wird nicht deutlich, was sie damit meint, dass die Jungen aus ihrer Sicht nicht wirklich mit dem Schmuck gespielt hätten, da sich die Jungen über längere Zeit mit dem Schmuck beschäftigt und mit mir Verarzten gespielt haben.

Alles in allem wird rekonstruierbar, dass Katrin die vermeintlich situative Relevanz von Geschlecht auf Ebene der Kinder ansiedelt und durch ihre Kommentierung wie cool für dich auch mir als Interessensfokus zuordnet. Sie als Fachkraft tritt lediglich als unbeteiligte Beobachterin kindlicher Aktionen auf, der nicht bewusst zu sein scheint, dass sie durch ihre nachträgliche Kommentierung die geschlechtlichen Relevanzsetzungen im Hinblick auf Jungen und Schmuck und damit das Thema Geschlecht selbst im Sinne von Stereotypen einbringt. So ist es ,gerade konstitutiv für das selbstverständliche und, wenn man so will, störungsfreie Ablaufen kultureller Praktiken [ist], daß die Akteure nicht explizit wissen, wie sie tun, was sie tun" (Kelle 2000: 120).

\section{Das Potenzial ethnographischer Perspektiven auf Geschlecht im Kontext von professionellem Handeln}

Friebertshäuser und Panagiotopoulou (2009) definieren „Ethnographische Feldforschung" als eine, Forschungstradition, die Menschen in ihrem Alltag untersucht, um Einblicke in ihre Lebenswelten zu erlangen „sowie ihre Sinndeutungen und Praktiken kulturanalytisch zu erschließen" (ebd.: 301). Laut Hitzler (2011) steht Ethnographie dabei für ein „Forschungsprogramm, das darauf abzielt, andere Lebensweisen, Lebensformen, Lebensstile sozusagen, von innen' her zu verstehen, d.h. ,fremde Welten' auf ihren Eigen-Sinn hin zu erkunden (...). Insofern (...) ist damit folglich ein Programm anzuzeigen, das empirisch stark deskriptiv orientiert ist - nämlich eben an den Erfahrungen, die Menschen machen" (ebd.: 48). Hier zeigt sich eine deutliche Abkehr von normativen Vorgaben und präskriptiven Setzungen, da es vielmehr ein wesentliches Anliegen ethnographischer Studien ist, an das Relevanzsystem der Akteur*innen des beobachteten Feldes anzuknüpfen, und das jenseits von Wirksamkeitsansprüchen und Outputorientierung. ${ }^{3}$ Meseth (2011) benennt das Potenzial einer ethnographischen Perspektiveinnahme, da sie ermöglicht, eine kritische Distanz „,zur Aufgabenstruktur moderner Pädagogik“ einzugehen.

3 Für den frühpädagogischen Bereich sind v.a. Peter Cloos ` Arbeiten zu nennen, die auf das Potenzial ethnographischer Zugänge in Professionalisierungszusammenhängen verweisen. 
Denn da Pädagogik ,,im Dienste des Subjektes Bildung fördern, Lernen ermöglichen, helfen, beraten oder erziehen soll“, bleibt sie „häufig in der normativen Struktur ihres Gegenstandes befangen“ (ebd.: 178). Auch Kelle (2004) sieht in einer notwendigen Distanzierung von normativen Vorgaben das Potenzial dieses empirischen Zugangs, denn ,[d] as Verhältnis von Ethnographie und Pädagogik kann zunächst so charakterisiert werden, dass die Ethnographie ,pädagogische Kulturen' nicht unmittelbar in Hinblick auf eine Evaluation der Theorie-Praxis-Vermittlung und Erreichung normativer Ziele fokussiert, sondern gewissermaßen neutraler, als kulturelle Praxis, deren Verfahren, symbolische Ordnungen sowie institutionelle Arrangements es - auch jenseits pädagogischer Absichten - detailliert und in situ zu erforschen gilt, um letztlich pädagogische Praxis weiter zu entwickeln“ (ebd.: 638). Auf Basis der ethnographischen Analysen der doing gender-Prozesse wird daher in Kubandt (2016) der Vorschlag unterbreitet, gängige Perspektiven auf Geschlecht sowohl empirisch, theoretisch als auch pädagogisch um einen deskriptiven Zugang zu ergänzen. Dies kann auch professionstheoretische Zusammenhänge produktiv ergänzen. Denn die Schwierigkeit ist, dass normativ geprägte Sichtweisen auf Geschlecht eine deutliche Verkürzung dessen darstellen, wie unterschiedlich Geschlecht in elementarpädagogischen Kontexten zum Thema werden kann. Es liegt in der Natur der Sache, dass einseitige Ausrichtungen - egal ob positiv konnotiert als anzuerkennende Querschnittsdimension oder negativ markiert als machtrelevante Ungleichheitskategorie - andere mögliche Perspektiven auf Geschlecht unterbeleuchtet lassen.

Die Auszüge der Studie zeigen, dass gerade alltägliche Interaktionen mit Herausforderungen im Hinblick auf professionelles Handeln verbunden sind: trotz der Bereitschaft der Fachkräfte, geschlechtergerecht zu handeln, werden geschlechtliche Stereotypisierungen während der Interaktionen nur bedingt wahrgenommen und nicht als solche reflektiert (vgl. auch Kubandt 2015). Demnach finden sich blinde Flecke seitens der Fachkräfte im Hinblick auf ihren unbewussten Umgang mit der Geschlechterthematik. Diese blinden Flecke zeigen sich allerdings auch in evidenzorientierten Perspektiven auf pädagogische Praxis. So kritisieren Hoffmann et al. (2014), dass alltägliche Interaktionen im Kontext von Professionalisierungsdebatten tendenziell unterbeleuchtet bleiben. Hier greift erneut das produktive Potenzial von ethnographischen Perspektiveinnahmen, die auch die Kontingenz und Nicht-Planbarkeit alltäglicher Interaktionen aufzeigen können. Wird dies nicht berücksichtigt, besteht die Gefahr, dass zwar analog der Aussage der Fachkräfte „wir behandeln alle gleich" die Ansprüche im Reden als realisiert zum Thema werden, sich an alltäglicher Praxis jedoch nichts ändert und es sich im Sinne Wetterers (2003) lediglich um eine rhetorische Modernisierung handelt. Anhand der aufgeführten Beispiele zeigt sich, dass es zentral ist, auch alltägliche Interaktionen zu berücksichtigen, wenn es um Fragen des geschlechtergerechten Handelns im pädagogischen Alltag geht (vgl. ausf. Kubandt 2018). So plädiert Karsten 
(2017) nicht ohne Grund dafür, die Diskontinuitäten des pädagogischen Alltags aufgrund seiner Unvorhersehbarkeiten noch stärker in Professionalitätsdebatten zu berücksichtigen. Denn dass berufliches Handeln nicht standardisierbar ist, ist ein zentrales Alleinstellungsmerkmal der personenbezogenen Dienstleistungsberufe (vgl. ebd.). Folglich bleibt der pädagogische Alltag stets unvorhersehbar, weshalb alltägliche Prozesse immer neu zu analysieren und zu reflektieren sind (vgl. Kubandt 2018). Bereits seit längerem fordern RabeKleberg (1993) und Karsten (2017) daher, dass Professionalität im Elementarbereich als Bereitschaft und Fähigkeit von Fachkräften aufgefasst werden sollte, die Ungewissheiten des eigenen Handelns zu ertragen und immer wieder neu zu reflektieren. Folgt man hingegen einem engen Kompetenzverständnis, wie es im Zusammenhang mit Wirksamkeitsanforderungen und Outputorientierung z.T. der Fall ist, zeigen sich deutliche Marginalisierungen der mit einem geschlechtergerechten Handeln einhergehenden Herausforderungen. Riegel (2013) verweist im Kontext von Ansprüchen an Diversity-Kompetenz auf mögliche Verkürzungen:

„ein solcher Kompetenz-Begriff [impliziert] die Vorstellung eines autonomen Subjekts sowie die ,Souveränität‘ und ,Unangreifbarkeit‘ von professionellem Handeln. Darin liegt auch das Versprechen einer gewissen Machbarkeit, d.h., wenn man sich die entsprechenden Kompetenzen aneignet, dann klappt der Umgang mit den Unterschieden auch. Dabei wird das Widersprüchliche von Handeln in einer durch Widersprüche geprägten Gesellschaft vernachlässigt, ebenso das eigene soziale Positioniert Sein und Involviert Sein in diese Verhältnisse“ (ebd.: 188).

Meyer (2018) formuliert Konsequenzen, wenn die Herausforderungen und Komplexitäten einer Realisierung von Ansprüchen unterschlagen werden und nicht transparent diskutiert wird, was ein angemessener Umgang mit sozialen Differenzen letztlich beinhaltet:

„Die pädagogische Bezugnahme auf soziale Differenz [...] wird damit für Fachkräfte zu einer Gratwanderung mit verbundenen Augen. Unter diesen Voraussetzungen ist das Scheitern genauso vorprogrammiert wie das Bild einer defizitären Praxis, die den gestellten Anforderungen nie voll und ganz gerecht wird“" (ebd.: 289).

Nimmt man die Komplexitäten und Herausforderungen hingegen ernst, dann ist es nicht verwunderlich, dass Praktiker*innen in den Einrichtungen verunsichert sind und die Ansprüche scheitern (vgl. Kuhn 2013; Kubandt 2016). Die mögliche Brückenfunktion von ethnographischen Perspektiveinnahmen im Hinblick auf Theorie-Praxis-Verhältnisbestimmungen in Professionalitätsdiskursen ergibt sich dann daraus, dass ethnographische Zugänge speziell die Logiken des Feldes in den Blick nehmen. In Meyer/Kubandt (2016) wurde daher für Geschlechterdebatten vorgeschlagen, Praktiker*innen ethnographische Datenmaterialien als Reflexionsfolien zur Verfügung zu stellen, um Fachkräften die Möglichkeit zu bieten, auch jenseits eines akuten Handlungsdrucks pädagogische Alltagspraxis in all seinen Komplexitäten in den Blick nehmen zu 
können. Ein damit verknüpftes Ziel wäre es, ein weniger hierarchisch gedachtes Verhältnis zwischen Wissenschaft und Praxis bzw. Disziplin und Profession zu ermöglichen, als es in wirksamkeitsorientierten Zugängen üblich ist. Die ethnographisch rekonstruierten Wissensbestände aus der Praxis selbst können dabei als Denk-, Reflexions- und Handlungsgrundlagen für eine wissenschaftlich informierte und geschlechterreflektierte professionelle Praxis dienen, und auf diese Weise auch als Brücke zwischen kompetenztheoretischen, strukturtheoretischen und berufsbiographisch orientierten Ansätzen fungieren.

\section{Literatur}

Diehm, Isabell/Kuhn, Melanie/Machold, Claudia (2013): Ethnomethodologie und Ungleichheit? Methodologische Herausforderungen einer ethnographischen Differenzforschung. In: Budde, J. (Hrsg.): Unscharfe Einsätze. (Re-)Produktion von Heterogenität im schulischen Feld. Neue Perspektiven auf Heterogenität. Wiesbaden: VS Verlag, S. 29-51. https://doi.org/10.1007/978-3-531-19039-6_2

Friebertshäuser, Barbara/Panagiotopoulou, Argyro (2009): Ethnographische Feldforschung. In: Friebertshäuser, B./Langer, A./Prengel, A. (Hrsg.): Handbuch Qualitative Forschungsmethoden in der Erziehungswissenschaft; 2. überarb. Aufl. Weinheim: Beltz Juventa, S. 301-322.

Hitzler, Ronald (2011): Ethnografie. In: Bohnsack, R./Marotzki, W./Meuser, M. (Hrsg.): Hauptbegriffe Qualitativer Sozialforschung. Opladen: Budrich, S. 48-51.

Hoffmann, Hilmar/Kubandt, Melanie/Lotte, Josefin/Meyer, Sarah/Nolte, David (2014): Professionelle Praxis im Spannungsfeld von evidenzbasiertem Wirken und Handeln in unsicheren Situationen - empirische Plausibilisierungen und Transferperspektiven. In: Fröhlich-Gildhoff, K./Nentwig-Gesemann, I./Neuß, N. (Hrsg.): Forschung in der Frühpädagogik Bd. 7. Freiburg: Verlag FEL, S. 47-81.

Karsten, Maria-Eleonora (2017): Gender-Mainstreaming in der Sozialpädagogik. In: Otto, H.U./Thiersch, H. (Hrsg.): Handbuch Soziale Arbeit: Grundlagen der Sozialarbeit und Sozialpädagogik. München: Reinhardt Verlag, S. 492-498.

Kelle, Helga (2000): Das ethnomethodologische Verständnis der sozialen Konstruktion der Geschlech-terdifferenz. In: Lemmermöhle-Thüsing, D./Fischer, D./Klika, D./Schlüter, A. (Hrsg.): Lesarten des Geschlechts. Zur De-Konstruktionsdebatte in der erziehungswissenschaftlichen Geschlechterforschung. Opladen: Budrich, S. 116-132.

Kelle, Helga (2004): Ethnographische Ansätze. In: Glaser, E./Klika, D./Prengel, A. (Hrsg.): Handbuch Gender und Erziehungswissenschaft. Prof. Doris Knab zum 75. Geburtstag. Bad Heilbrunn: Klinkhardt, S. 636-650.

Knapp, Gudrun Axeli (2001): Kein Abschied von Geschlecht. Thesen zur Grundlagendiskussion in der Frauen- und Geschlechterforschung. In: Hornung, U./ Gümen, S./Weilandt, S. (Hrsg.): Zwischen Emanzipationsvision und Gesellschaftskritik. (Re)Konstruktionen der Geschlechterordnung in Frauenforschung, Frauenbewegung, Frauenpolitik. Münster: Westfälisches Dampfboot, S. 78-86. 
Kubandt, Melanie (2015): „Oh man, was hab ich denn jetzt gesagt?!“ Doing gender von Fachkräften in der Kindertageseinrichtung. In: Hoffmann, H./Borg-Tiburcy, K./Kubandt, M./ Meyer, S./Nolte, D. (Hrsg.): Alltagspraxen in der Kindertageseinrichtung. Annäherungen an Logiken in einem expandierenden Feld. Weinheim/Basel: Beltz Juventa, S. 88-120.

Kubandt, Melanie (2016): Geschlechterdifferenzierung in der Kindertageseinrichtung - eine qualitativ-rekonstruktive Studie. Opladen: Budrich.

Kubandt, Melanie (2018): Geschlechterkonstruktionen in der Kindertageseinrichtung ethnographische Perspektiven auf den elementarpädagogischen Alltag als Beitrag zur Professionalisierung der Sozialen Arbeit. In: Aghamiri, K./Reinecke-Terner, A./Streck, R./Unterkofler, U. (Hrsg.): Doing Social Work. Ethnografische Forschung als Theoriebildung. Opladen: Budrich, S. 151-171.

Kuhn, Melanie (2013): Professionalität im Kindergarten: Eine ethnographische Studie zur Elementarpädagogik in der Migrationsgesellschaft. Wiesbaden: Springer VS. https://doi.org/10.1007/978-3-531-19158-4

Machold, Claudia (2015): Kinder und Differenz. Eine ethnographische Studie im elementarpädagogischen Kontext. Wiesbaden: Springer VS.

Meseth, Wolfgang (2011): Erziehungswissenschaft - Systemtheorie - Empirische Forschung. Methodologische Überlegungen zur empirischen Rekonstruktion pädagogischer Ordnungen. In: Zeitschrift für Qualitative Forschung (ZQF), Jg. 12, Heft 2, S. 177-197.

Meyer, Sarah (2015): Differenzierungen im Spannungsfeld zwischen Reproduktion, institutioneller Selbstrepräsentation, Einpassung und Modifikation. In: Hoffmann, H./Borg-Tiburcy, K./Kubandt, M./Meyer, S./Nolte, D. (Hrsg.): Alltagspraxen in der Kindertageseinrichtung. Annäherungen an Logiken in einem expandierenden Feld. Weinheim/Basel: Beltz Juventa, S. 120-154.

Meyer, Sarah (2018): Soziale Differenz in Bildungsplänen für die Kindertagesbetreuung. Eine diskursiv gerahmte Dokumentenanalyse. Wiesbaden: Springer VS. https://doi.org/10.1007/978-3-658-20239-2

Meyer, Sarah/Kubandt, Melanie (2016): „Und was heißt das jetzt für die Praxis?“ Zur Frage der Praxisrelevanz ethnographischer Forschungsergebnisse im Kontext von Geschlecht. In: Graff, U./Kolodzig, K./Johann, N. (Hrsg.): Ethnographie - Pädagogik - Geschlecht. Projekte und Perspektiven aus der Kindheits- und Jugendforschung. Wiesbaden: Springer VS, S. 121-137. https://doi.org/10.1007/978-3-658-07280-3 7

Niedersächsisches Kultusministerium (2005) (Ḧrsg.): Orientierungsplan für Bildung und Erziehung im Elementarbereich niedersächsischer Tageseinrichtungen für Kinder. Hannover.

Rabe-Kleberg, Ursula (1993): Verantwortlichkeit und Macht - Ein Beitrag zum Verhältnis von Geschlecht und Beruf angesichts der Krise traditioneller Frauenberufe. Bielefeld: USP International.

Rendtorff, Barbara (2017): Was ist eigentlich gendersensible Bildung und warum brauchen wir sie? In: Glockentöger, I./ Adelt, E. (Hrsg.): Gendersensible Bildung und Erziehung in der Schule. Waxmann Verlag: Münster, S.17-25.

Riegel, Christine (2013): Diversity-Kompetenz? - Intersektionale Perspektiven der Reflexion, Kritik und Veränderung. In: Faas, S./Bauer, P./Treptow, R. (Hrsg.): Kompetenz, Performanz und soziale Teilhabe. Sozialpädagogische Perspektiven auf ein bildungstheoretisches Konstrukt. Wiesbaden: VS Verlag, S. 183-195. 
Wetterer, Angelika (2003): Rhetorische Modernisierung: Das Verschwinden der Ungleichheit aus dem zeitgenössischen Differenzwissen. In: Knapp G.-A./ Wetterer, A. (Hrsg.): Achsen der Differenz. Gesellschaftstheorie und feministische Kritik II. Münster: Westfälisches Dampfboot, S. 286-320. 